Psychological Medicine, 1978, 8, 5-8

Printed in Great Britain

\title{
EDITORIAL
}

\section{The future of alcoholism: a commentary on the Rand Report ${ }^{1}$}

The future of alcoholism has looked gloomy for a while now. The signs, in the form of drunkenness offence and alcoholism hospital admission statistics, as well as figures for national alcohol consumption, are that alcohol problems are on the increase (these signs may be unreliable but they are about all we have), and until recently there have been few advances in knowledge which have practical value. The enthusiasm which accompanied the greater acceptance of Alcoholism-as-a-disease 2 or 3 decades ago has waned, and if we are honest with ourselves we have to admit that we are impotent to prevent alcoholism occurring and have recorded little success in treating it. It is against this background that the Rand Report has to be considered.

\section{THE REPORT AND ITS FINDINGS}

The Report's proper title is Alcoholism and Treatment; its authors are David Armor, Michael Polich and Harriet Stambul, and it was published by the Rand Corporation in the United States in June 1976. Rand had been engaged to analyse data collected by the National Institute of Alcohol Abuse and Alcoholism (NIAAA) from clients attending their Alcoholism Treatment Centres which had been set up in recent years, as a result of a large injection of public money, in a number of towns across the United States.

The Report was lengthy, detailed and highly statistical, but it reached 2 particular conclusions that went against much conventional thinking on the subject and which provoked a great deal of angry comment. The first was that many clients give up drinking excessively with only very minimal treatment or with next to no treatment at all. The second, even more provocative, conclusion was that many who did give up excessive drinking w'ere found later to be drinking 'normally' and not abstaining totally as the conventional wisdom dictates is necessary. It was this conclusion that elicited most reaction and which is especially examined in this editorial.

\section{CRITICAL REACTION TO THE REPORT}

The US National Council on Alcoholism issued a press release on 1 July 1976 and there have been many other critical comments since. Some of these are fair, to the point, and indisputable. The first is that this one report, among many that have appeared in the last few years on the same topic, received much wider and more public dissemination and should have been published in the scientific press in the normal way so that its findings could stand the test of informed and detailed criticism before the public at large were exposed to them. Another was that data which were analysed were obtained from only a proportion of NIAAA Treatment Centres, and that even then follow-up, both at 6 months (only $21 \%$ ) and at 18 months (62\%), was very far from complete. Furthermore, followup data were based upon verbal accounts given by clients alone, not supplemented by objective data or accounts by family members or others, and hence must suffer from the under-reporting of drinking which is known to affect such self-accounts.

Other criticisms of the report are more debatable but have a familiar ring to them. One claim is that many members of the sample were not suffering from alcoholism at all, but were merely 'problem drinkers' who were not physically dependent upon alcohol. In fact, Armor et al. (1976) made separate analyses for those clients whom they define as 'definitely alcoholic' (roughly three-quarters) and the remainder. To be included in the former category at least one of the following criteria had to be met: (1) drinking more than 12 ounces of ethanol on a typical drinking day (equivalent to a little over a

${ }^{1}$ Address for correspondence: Dr Jim Orford, Exe Vale Hospital, Woodwater Lane, Digby, Exeter EX2 7EY. 
full bottle of spirits, or roughly 12-15 pints of beer); (2) one or more episodes of tremor; (3) frequent episodes of at least 3 of the following 6 'symptoms'-tremor, blackout, missing meals, morning drinking, being drunk, missing work. Vital though it is to describe carefully the characteristics of clients who contributed to a report of this importance, questions about who is, and who is not, an 'alcoholic' are generally beside the point, however. Like the clientele of any alcoholism treatment programme anywhere, the Rand sample was a fair mixture of people with varying degrees of alcohol problem, and any dichotomous categorization must be arbitrary. There is no absolute definition of 'alcoholism' and certainly no clear division in practice between those who are physically dependent upon alcohol and those who are not. What is irrefutable is that a proportion of people with drinking problems sufficiently severe to warrant referral to a specialist treatment agency were found at follow-up to be drinking in a limited fashion.

A further, and again largely unwarranted, criticism is that what Rand defined as 'normal drinking' was not normal at all. In fact, to be included in the normal drinking category at follow-up a client had to report drinking a daily average of less than 3 ounces of ethanol (in fact the average "average" for the normal drinking group was merely 0.7 ounces), and a typical quantity on a drinking day of less than 5 ounces, and an absence of tremor, and an absence of any one of the 6 symptoms mentioned above (all with reference to the previous month). The word 'normal' implies a comparison with others in the general population, and of course it is true that many people drink only very occasionally or not at all. The word 'limited' is perhaps more accurate, and its substitution for the word 'normal' in no way alters the report's conclusions or their importance.

A third, familiar, and quite fallacious argument is that the follow-up limited drinking rate was small and of no practical significance. In fact, at 6 months limited drinkers represented $12 \%$ of the follow-up sample, and at 18 months $22 \%$. These figures are very much in line with the types of figures reported in numerous other studies that have been published in scientific journals in the last decade or so (e.g. Gerard \& Saenger, 1966). In any case, to measure their importance these percentages should obviously be compared with the percentages of clients who are successfully abstaining totally from alcohol at follow-up. The latter are also usually a minority. In fact, Armor et al. (1976) report unusually high follow-up abstinence rates (56\% at 6 months, and $45 \%$ at 18 months), perhaps because they based their figures upon self-reports, and upon accounts merely of the previous month. From the range of previously published findings, it is clear that limited drinkers usually represent a substantial minority, and occasionally even a majority, among people who have been treated or counselled for drinking problems, even when everyone was advised to abstain totally (as was generally the case at the NIAAA clinics).

Then there is the criticism that 18 months is insufficient time to determine whether limited drinking lasts, the assumption being that relapse is inevitable sooner or later. To be fair, 18 months is an uncommonly long follow-up period for studies of this kind, and it is usually found that most relapses occur in the first 6 months (e.g. Hunt \& Matarazzo, 1970). In any case, there is no reason why prognosis should be more pessimistic for limited drinking than for abstinence. Indeed, an important finding of the Rand analysis was that slightly fewer 6-month limited drinkers than 6-month abstainers had relapsed by 18 months (13\% versus $18 \%$ - although it is true to say that a high proportion of abstainers had abstained for at least 6 months by the later follow-up, and figures are not quoted which would indicate how many people kept up limited drinking for a comparable period of time.

Finally, and this is quite unfair, Armor and his colleagues are accused of irresponsibly overstating their conclusions. Nothing could be further from the truth. The following quotation is typical of their caution. It is, they say, 'premature to endorse or advocate a policy of normal drinking for alcoholics ... data are ... not adequate to establish beyond question the long term feasibility of normal or "controlled" drinking among alcoholics. . . . Thus we do not make any policy recommendation' (p. 140). 


\section{TAKING RAND SERIOUSLY}

There is one very good reason why Rand should not be dismissed off-handedly. If the results had been out of line with other evidence we should naturally be suspicious because of the study's limitations of method. On the contrary, because their results are so much in line with evidence that has been accumulating in the literature, the results can hardly be ignored and attributed merely to the study's methodological shortcomings.

First of all, there is now a great deal of evidence to suggest that the precise nature of treatment, as well as the quantity of treatment received, is relatively unimportant (e.g. Willems et al. 1973; Orford \& Edwards, 1977). Rand's conclusion that "perhaps the crucial ingredient in treatment success is not really treatment at all, but rather the person's decision to seek treatment and remain in treatment' (p. 96), would now be echoed by many. In our efforts to help people with serious drinking problems we have been looking in quite the wrong direction. Instead of trying to understand, and encourage, people's own decisions about behaviour, we have been attempting to administer to them in a pseudo and misleading 'treatment of disease'-like fashion.

In the case of the controversial normal or limited drinking findings, the Report also repeats previous work by others, with much larger numbers than usual (over 2000 clients were represented in the 6-month follow-up data, and over 500 in the 18-month)-although surprisingly the authors of the report have been criticized for their small numbers! Not only are the proportions of people drinking in a controlled way at follow-up very comparable to those found in other research, but many of the detailed findings also replicate previous work and therefore strengthen confidence in their validity. For example, the longer the follow-up the more people who are found to be successfully limiting their drinking rather than abstaining (e.g. Kendell, 1965; Orford et al. 1976). Of the greatest importance, however, are the Rand Report's indications of those who are most likely to be able to control or limit their drinking after having a serious drinking problem. The strongest factor predicting the relative proportions of controlled drinkers and abstainers at follow-up was affiliation with Alcoholics Anonymous. Abstainers far outnumbered limited drinkers among clients who had been regularly attending A.A., while limited drinkers outnumbered abstainers among clients who had no A.A. attendance in the previous year. Furthermore, even though different treatment clinics vary little in their overall success rate, there were intriguingly large differences in the proportions of successful clients who were drinking in a limited way at follow-up at the different centres (Gerard \& Saenger, 1966 , found the same). These results strongly suggest that the orientation of the counsellors to whom a client is exposed is a crucial factor in determining the nature of the outcome (abstinence or limited drinking) but not the overall success or failure of the outcome.

Of special significance, because of the practical implications, were differences between those clients who were rated as 'definitely alcoholic' when they first came to the clinic, and the others. While $16 \%$ of the former are described as drinking 'normally' at 18 months follow-up, the percentage for those with less definite symptoms was 36 . Abstainers outnumbered limited drinkers by almost 3 to 1 among former 'definite alcoholics', while abstinence and limited drinking were almost equally likely among the remainder. Again this confirms findings in this country: in a study of 100 men followed up for 2 years, abstinence success was associated with having 4 or more of the following symptoms at intake: morning drinking, tremor, morning nausea, 'loss of control' over the amount drunk, passing out, secret drinking and hallucinations. Controlled drinking success was associated with having fewer symptoms (Orford et al. 1976). Although there is little justification for splitting people with drinking problems sharply into 'alcoholics' and the rest, it may be that there are important indications of the degree of alcohol dependence which indicate whether regaining control over drinking, and the resumption of limited drinking, are possible. 


\section{THE FUTURE OF ALCOHOLISM}

The evidence that some people with drinking problems sufficiently severe to warrant referral to a specialist can nevertheless drink in a limited way within a year or two is now overwhelming. There are numerous studies showing that this happens even if people are advised to abstain totally; and that when treatment programmes are set up with the explicit intention of advising some clients to limit their drinking rather than to abstain (e.g. Sobell \& Sobell, 1973), overall treatment results are just as good as those achieved by abstinence-oriented programmes. The only difference is that the proportion of limited drinkers is greater, and the proportion of abstainers correspondingly smaller. Even then, a proportion of clients choose to abstain, and indeed almost all therapists and counsellors continue to advise many clients to abstain. In my view this is quite correct. The greatest hope for the future of alcoholism is that of having more than one goal on offer, and of being able to advise people rationally on which goal would suit them best. As the authors of the Rand Report state, "future bio-medical and behavioural research must directly address the question of possible . . differences between alcoholics who can return to and maintain normal drinking and alcoholics who cannot' (p. 140). Meanwhile, the type of person who might be best advised to try and limit their drinking may te the person who at some time in his or her life had established a fairly normal pattern of limited drinking (by no means all people with serious drinking problems have ever done this), who has experienced at most mild or irregular withdrawal symptoms, who continues to retain some degree of control over drinking sometimes, who has not established a strong affiliation with an abstinence-oriented organization or clinic, and who expressed a preference for limited drinking rather than abstaining.

Some critics of Rand believe that the evidence should be suppressed because it could have tragic consequences for individual drinkers tempted to try and drink again. The real tragedy would be if prejudice and undue caution prevented an exploration of alternative drinking goals. Without such exploration, alcoholism has a cheerless future. However, no one would advise a person who has already successfully attained abstinence to try limited drinking without good reason.

JIM OR FOR D

\section{REFERENCES}

Armor, D. J., Polich, J. M. \& Stambul, H. B. (1976). Alcoholism and Treatment. The Rand Corporation: Santa Monica, California.

Gerard, D. L. \& Saenger, G. (1966). Out-patient Treatment of Alcoholism: a Study of Outcome and its Determinants. University of Toronto Press: Toronto.

Hunt, W. A. \& Matarazzo, J. D. (1970). Habit mechanisms in smoking. In Learning Mechanisms in Smoking (ed. W. A. Hunt), p. 85. Aldine: Chicago.

Kendell, R. E. (1965). Normal drinking by former alcohol addicts. Quarterly Journal of Studies on Alcohol 26, 247-257.
Orford, J. \& Edwards, G. (1977). Alcoholism: A Comparison of Treatment and Advice, with a Study of the Influence 0 . Marriage. Oxford University Press: London.

Orford, J., Oppenheimer, E. \& Edwards, G. (1976). Abstinence or control: the outcome for excessive drinkers two years after consultation. Behaviour Research and Therapy 14, 409-418.

Sobell, M. B. \& Sobell, L. C. (1973). Individualized behaviour therapy for alcoholics. Behaviour Therapy 4, 49-72.

Willems, P., Letemendia, F. \& Arroyave, F. (1973). A followup study of short- and long-term inpatient treatment of alcoholics. British Journal of Psychiatry 122, 637-648. 